\title{
Los distritos industriales italianos y su repercusión en el desarrollo de las pequeñas y medianas empresas ${ }^{1}$
}

\author{
Enrique Sánchez Slater ${ }^{2}$
}

\begin{abstract}
RESUMEN
El artículo se enfoca sobre las ventajas, derivadas de las economías externas, que obtienen las pequeñas y medianas empresas pertenecientes a los distritos industriales italianos o a un sistema productivo local y su vinculación con el concepto de desarrollo local en el territorio donde se produce la aglomeración. Para ello se reconstruye la génesis histórica del concepto del distrito desde su autor, Alfred Marshall y en como estas ideas fueron rescatadas por Giacomo Becattini adaptándolas a la realidad industrial italiana a mediados de los años setenta. El modelo es de especial relevancia en la escuela económica industrial puesto que ha roto con el viejo paradigma de la gran empresa verticalmente integrada.
\end{abstract}

Palabras clave: Distrito industrial, economías externas, desarrollo local, territorio.

\begin{abstract}
This article focuses on the advantages derived from the external economies that the small and medium companies belonging to the Italian industrial districts can obtain and its relationship with the concept of local development in the territory where the agglomeration is produced. For the above, a historical genesis of the district concept, from the author Alfred Marshall, is reconstructed. There is also an analysis of how these ideas were rescued by Giacomo Becattini adapting them to the Italian industrial reality during the seventies. The model is of special relevancy in the industrial economic school since it has broken with the old paradigm of the vertically integrated company.
\end{abstract}

Key words: Industrial district, external economies, local development, territory.

La discusión y la polémica sobre el papel de la pequeña empresa como vector de desarrollo económico continúan hasta nuestros días.

De hecho, algunos economistas y sociólogos de los años cincuenta y sesenta consideraban a la pequeña empresa como un

1 Artículo recibido el 20 de diciembre de 2007 y aceptado el 17 de abril de 2008.

2 Investigador doctoral en el Programa de Doctorado en Economía y Gestión de la Innovación y Política Tecnológica, departamento de economía y lugar donde se conjugaban el retraso, tanto a nivel tecnológico como de innovación. Sin embargo, esta lectura empieza a cambiar a mediados de los setenta debido al aumento de una demanda personalizada y a la rapidez en el cambio técnico, factores que ponen en crisis los métodos de producción construidos sobre la base del modelo

organización de empresas, Universidad Autónoma de Madrid (España) - Investigador asociado al Instituto de Desarrollo Local y Regional, IDER, Universidad de la Frontera, Temuco (Chile). E-mail: esanchez@ufro.cl 
Fordista (Becattini, 2000; Piore \& Sabel, 1984).

El caso de los distritos industriales italianos ha abierto un nuevo camino en la organización territorial de la producción, sustentada en gran parte por la pequeña y mediana empresa. La densidad en el territorio y el número de empresas que ahí se constituyen dan origen a economías externas de escala (Schmitz, 1999), las cuales han beneficiado al desarrollo y crecimiento de las PYMES italianas pertenecientes a un distrito industrial o a un sistema productivo local.

Además de la importancia que cobra el territorio, se deben añadir otras variables que son de especial relevancia como son: la división del trabajo ${ }^{3}$, la cooperación entre las empresas, la interacción con la comunidad local que habita y trabaja en dicho lugar, y el papel de las instituciones en garantizar las reglas del juego ${ }^{4}$ (North, 1994).

El distrito industrial representa un modelo dinámico de organización de la producción, de absorción y reproducción del conocimiento y de recursos humanos. Los elementos clave en la atención a los procesos de transformación de los sistemas productivos locales son la innovación y el cambio, conformando, a su vez, los pilares clave del desarrollo de la economía italiana basada en empresas de pequeña dimensión.

\section{Una excepción al paradigma fordista de la producción en masa: el dualismo industrial}

La historia señala que la producción a pequeña escala realizada fundamentalmente por artesanos cualificados no es un fenómeno nuevo, ni tampoco tiene su génesis en

\footnotetext{
3 En relación a la especialización de la pequeña empresa por fases de producción en torno a una industria principal.

4 El papel de las instituciones es fundamental en los distritos industriales a la hora de establecer las reglas del juego, garantizando la aplicación de los contratos y definiendo y tutelando los derechos de propiedad. Por otro lado, juega un papel relevante en el aspecto económico, al rebajar los costes derivados de la actividad comercial y de cooperación.
}

Italia. Ya desde los tiempos de la revolución industrial (a mediados del siglo XVIII) existía una producción artesanal que basada sobre la idea de que la máquina y los procesos podían incrementar la habilidad manual, permitiendo al trabajador explotar su propia capacidad para fabricar una mayor cantidad de productos (Piore \& Sabel, 1984).

La otra forma de desarrollo tecnológico era la producción en masa o en serie (a fines del siglo XIX), que se basaba sobre el principio de que el coste de fabricación de un producto determinado podía ser notablemente reducido si las máquinas sustituían el trabajo humano. Su finalidad era descomponer cada tarea manual, en fases simples, donde cada una de ellas podía ser efectuada de manera más rápida y precisa. Para ello era necesaria la creación de una máquina especializada, impactando de esta forma los métodos de producción a gran escala.

La producción en serie fue la dominante en los países avanzados hasta mediados de los años setenta del siglo pasado. Sin embargo, la fragmentación de los mercados y el cambio en los gustos de los consumidores puso en crisis el tipo de producción estandarizada enfocada a grandes masas de consumidores.

El cambio en los gustos de los consumidores, es decir, de quien demanda los productos, se extiende desde varias capas medias en los países avanzados, y son en definitiva, aquella clase que busca el Standard of Confort ${ }^{5}$. La superación de dicho estándar crea las condiciones para el nacimiento de nuevos núcleos de necesidades de alto contenido social, basándose en la calidad de los bienes, lo que provoca demandas muy variables en la búsqueda de satisfacer productos diferenciados y personalizados (Becattini, 2002: 19).

En la Italia del milagro económico (a inicios de los sesenta), dentro de un mundo occidental en gran expansión se vuelven a crear unas condiciones análogas a las de muchos centres of specialized británicos

5 Becattini (2002) contrapone el concepto Standard of confort al de standar for living de Alfred Marshall (1961). 
afectados por la primera gran expansión que siguió a la revolución industrial, en ambos casos un amplio y desigual crecimiento de la renta concentra esta en manos de un gran núcleo de clases medias que, alcanzando standar of confort del momento, se dedica a la búsqueda de productos y servicios más diferenciados y personalizados que aporten sensaciones nuevas y prestigio social. Estas teorías del mundo real, señalan entre otras variables, el nacimiento o renacimiento ${ }^{6}$ de los distritos industriales.

\section{El concepto integral de desarrollo económico y su vinculación con el modelo del distrito industrial italiano}

El desarrollo económico consiste en crear, dentro de una economía local o regional, la capacidad necesaria para hacer frente a los retos y oportunidades que puedan presentarse en una situación de rápido cambio económico, tecnológico y social. No se trata solo de un simple incremento de la actividad y la productividad económica, sino que viene acompañado de transformaciones estructurales; el proceso implica profundas modificaciones y una evolución de sus estructuras hacia formas superiores.

Ahora bien, la conceptualización del desarrollo económico y lo que es más importante, su implantación, es diferente según la zona geográfica en la cual se lleve a cabo, por lo que habrá que estudiar las diferentes oportunidades y problemas de cada país, región y localidad en particular, surgiendo así el concepto de desarrollo local.

A lo largo de su evolución, el concepto de desarrollo se ha manifestado de múltiples formas, pudiendo hacer referencia a diferentes enfoques del mismo. Desde que Birch (1979) propuso su interpretación de la natalidad y mortalidad de las empresas, una parte creciente de la literatura sobre el desarro-

6 La teoría de los distritos industriales italianos tiene su origen en autores como Giacomo Becattini, quien se inspira y rescata aquellas teorías acerca de la organización de la producción de Alfred Marshall en la segunda mitad del siglo XIX, en Inglaterra.
Ilo trató de explicar el surgimiento y expansión de estas como una fuente destacada de progreso de las sociedades.

Posteriormente, al incorporar la dimensión espacial surgen tres nuevas aproximaciones al concepto de desarrollo:

- La interpretación estructural que concede una especial importancia a los factores estructurales y de dimensión (Gudgin, 1978);

- La teoría de la reestructuración regional (Markusen, 1985); y

- La teoría espacial al desarrollo (Becattini, 1979; Fuà, 1983).

Las dos últimas teorías ponen énfasis en los factores socioculturales del entorno y los de oferta-demanda en las decisiones de inversión y localización de las empresas, de ahí la importancia del territorio en términos de governance y la articulación de las instituciones para el desarrollo de las regiones y países.

Así entonces, a finales de los años setenta y comienzos de los ochenta surgen nuevas variantes a este modelo, como es el caso del enfoque de los distritos industriales italianos (Becattini, 1979; Bellandi, 1986), del Modelo de Industrialización Descentralizada (Fuà, 1988; Vázquez Barquero, 1988) o el de los Polos Tecnológicos.

\section{Las variables que conforman el modelo del distrito industrial italiano}

\section{El análisis de las economías externas y del distrito, según Alfred Marshall}

Ha transcurrido casi un siglo desde que Alfred Marshall precisó por vez primera, el concepto de economías externas. En el pasaje de Industry and Trade, el joven Marshall señala:

"Las economías (externas) de la producción a gran escala, no deben ser atribuidas a cualquier industria, puesto que ellas están en gran medida conectadas con grupos, a menudo grandes grupos de industrias entre las cuales existe una correlación" (Marshall, 1919: 188). 
Marshall identifica tres razones para la localización de la industria, sosteniéndose en las ventajas que se producen de las economías externas que son:

a) Pool de mano de obra: la concentración sectorial y geográfica de las empresas crea un pool de habilidades y especialización de la mano de obra que beneficia tanto al trabajador como a las empresas;

b) Proveedores especializados en imputs: Io que beneficia a las empresas que allí residen al encontrar un soporte especializado tanto en imputs como en servicios dentro del territorio;

c) Spillovers tecnológicos: que ayudan a la difusión y circulación de nuevas innovaciones, además de acumulación de Know How en dicho territorio.

Su visión de la economía externa se cumple dentro del cuadro teórico-empírico en referencia al distrito industrial italiano, condiciones que han sido verificadas. De hecho, las economías externas constituyen ventajas equivalentes a las economías internas de escala, así entonces las pequeñas empresas se podrían beneficiar de la concentración territorial, lo que se traduciría en una posibilidad de desarrollo inducido por las inversiones en una industria subsidiaria y por la elaboración de bienes necesarios a una industria regional.

\section{El territorio como variable de desarrollo}

Desde los tiempos de la revolución industrial en Inglaterra el territorio ha sido objeto fundamental en el análisis del desarrollo económico. En lo que concierne a los distritos italianos, la reflexión está centrada sobre la relación entre la producción y la organización productiva en un lugar determinado. Esto es, las relaciones de colaboración entre empresas, sistemas productivos, sistemas socioinstitucionales, movilidad de los trabajadores en la aglomeración de la producción, y el papel de las instituciones que intervienen directamente en el sustento del desarrollo y crecimiento de la empresa; hablamos de centros tecnológicos, centros de servicios especializados para la empresa, centros de formación profesional y agencias para el desarrollo local.

El territorio se vuelve competitivo no solo cuando se constituye en un sistema local, como es el caso de los distritos industriales, sino también cuando es capaz de afrontar la competencia del mercado, garantizando así una estabilidad económica, social y cultural (Giardello, 2006). Tal proceso puede dar lugar a diversas modalidades de desarrollo en la cual el distrito solo representa un prototipo. Dicho en otras palabras, los factores fundamentales del desarrollo están históricamente radicados en la realidad socialcultural y por tanto, no son fácilmente transferibles a otros territorios, y "esto es en definitiva que el desarrollo se manifiesta como un fenómeno social y no como un proceso exclusivamente técnico" (Garofoli, 1991: 74). Cada uno de ellos cuenta con su propia historia, por eso es importante determinar en cada caso factores tales como el pasado industrial y el capital social $^{7}$ de la zona en particular.

\section{La división del trabajo dentro del distrito y sus efectos en las empresas de pequeña dimensión}

La división y movilidad en el trabajo y las relaciones entre las empresas que se originan de la misma, son otro elemento fundamental del distrito industrial, el que la hace diferente de otros modelos de organización industrial, como por ejemplo los clusters ${ }^{8}$.

\footnotetext{
7 Son muchos los autores que hacen referencia a este concepto, "se refiere a los valores y las creencias que los ciudadanos conviven en cuestiones cotidianas, dando significado y motivo a cada tipo de norma y regla" (Maskell, 2000).

8 La división del trabajo es de menor intensidad en los clusters debido a un tipo de producción más estandarizada, a un menor contacto con el conjunto de la comunidad local, y a una relación más lejana con el mercado final. Esta situación se pudo observar en terreno en la visita realizada por el autor al sistema productivo local de la materia plástica en Varese (Lombardía), que según estudios de la Università degli studi d'Insubria (Varese), reúne características más afines a un cluster que a un distrito.
} 
Para señalar la importancia de la división del trabajo de forma más clara se citará un ejemplo. El ciclo de producción textil en Italia se divide en múltiples operaciones que van desde la producción de la fibra textil a la de productos finales para uso industrial y de consumo. Así entonces se identifican tres estadios en el proceso:

a) La producción de fibra y fila continua (hilatura);

b) La producción textil propia (el tejido); y

c) La producción del manufacturado (confección del producto final).

Cada una de ellas se subdivide, a su vez, en otras fases, por ejemplo la hilatura de fibra natural se caracteriza por los procesos de cardado, planchado e hilado. El tejido se divide en el tejido propiamente tal, el tejido a punto, etc.

Son varias las fases que intervienen en los procesos productivos antes de lograr el producto final. La distribución y división del trabajo debe estar entre los factores primordiales en los procesos de reestructuración adoptados en el área, con el objeto de proporcionar las combinaciones óptimas de los factores de producción en cada segmento del ciclo. La división del trabajo trae consigo una estrecha relación entre las pequeñas empresas que forman parte del proceso, aumentando los flujos de información y movilidad en el mercado del trabajo ${ }^{9}$ lo que implicará un know how añadido en el trabajador que permanezca en el área, ya que esta interdependencia entre las empresas es a nivel infrasectorial e intersectorial. Sin embargo, las repercusiones tendrán más efecto en aquellas relaciones de tipo horizontal, provocando una baja en los costes de transacción entre las empresas (Garofoli, 1991).

9 "Una de las ventajas de los distritos deriva de la capacidad y flexibilidad en el mercado del trabajo, elemento que se creará con la especialización productiva y la relación entre las empresas en un territorio circunscrito" Becattini et al. (2001: 129).

\section{La comunidad local y las instituciones}

En lo que concierne a la comunidad local, intervienen elementos sociológicos bastante fuertes y arraigados, se trata de un sistema bastante homogéneo de valores que se exprimen en términos de ética del trabajo, de la actividad, la familia (particularmente importante en Italia), de la reciprocidad. El sistema de valores que prevalece en el distrito se forma con en el tiempo, lo que a su vez constituirá un elemento importante para su continuidad.

En cuanto a las instituciones, las pequeñas y las grandes empresas, la demanda de regulación y bienes públicos es bastante heterogénea. Las grandes empresas deben invertir fuertemente en planificación de la producción. Tiende a operar en un horizonte temporal a largo plazo y debe anticipar las características de la demanda. Las empresas pequeñas, en cambio, basan su propia eficiencia en la valoración de los recursos técnicos, en la competencia del emprendedor, y en los limitados costes de transacción. Estas características le consienten una mayor movilidad de entrada y salida de los segmentos del mercado.

El modelo que representa la experiencia italiana se caracteriza por un bajo nivel de regulación central y por una iniciativa relevante de los sujetos institucionales intermedios. Se basa, en resumen, sobre la renuncia de la autoridad central a orientar el cambio estructural y la especialización sectorial de la industria, y a coordinar los procesos de concentración y reorganización que se realizan a través de inversiones directas (como en Francia) o con instrumentos de disciplina en la competencia, como ocurre en Gran Bretaña (Arrighetti y Seravalli, 1997).

Las instituciones intermedias, asimismo, han podido suplir, al menos en parte, la ausencia de iniciativa de la autoridad central a través de intervenciones de regulación sectorial o local. El acento institucional después de la Segunda Guerra Mundial se volcó en privilegiar la regulación descentralizada. Esta estrategia se ha desarrollado sobre la política de exención, que creó una cuota significativa 
de trabajo autónomo en las empresas de pequeña dimensión, esto es un régimen especial de gravamen fiscal, de simplificación de la producción administrativa y de agilidad crediticia. Estas intervenciones se han traducido en la reducción de las barreras de entrada y en general, en los costes de constitución de las empresas, facilitando así la absorción de la elevada oferta emprendedora que ha caracterizado las primeras fases del desarrollo económico italiano.

\section{La contribución de los sistemas productivos locales a la economía italiana}

Los distritos industriales han sido un pilar fundamental del desarrollo italiano desde la Segunda Guerra Mundial hasta hoy. Aunque bien es cierto que en la última década han debido afrontar problemas de crecimiento y competitividad, principalmente por la entrada de nuevos competidores (China,
India, México, Brasil), nadie puede poner en duda que han sido las vértebras de la infraestructura manufacturera italiana, sostenida fundamentalmente por la pequeña y mediana empresa especializada.

La cuota de mercado de Italia, es decir, la incidencia de las exportaciones italianas sobre el total del comercio mundial, creció de manera sistemática hasta los años noventa, como puede observarse en los Cuadros $N^{0} 1$ y 2.

Los datos muestran (Cuadro $N^{\circ} 2$ ) la importancia de los sectores tradicionales del made in Italy (textil-confección, muebles, cuero, calzado), sectores que en teoría, carecen de innovaciones tecnológicas importantes y además producen en pequeñas escalas debido a sus métodos de producción flexible ${ }^{10}$.

10 La producción de tipo flexible implica un mayor contacto con el cliente final de la cadena de valor.

\section{Cuadro $\mathrm{N}^{\circ} 1$}

CONTRIBUCIÓN DE LOS SISTEMAS LOCALES MANUFACTUREROS PYMES A LAS EXPORTACIONES NACIONALES POR ACTIVIDAD ECONÓMICA

\begin{tabular}{|l|c|}
\hline \multicolumn{1}{|c|}{ Tipo de industria } & $\begin{array}{c}\text { Contribución a las } \\
\text { exportaciones (\%) }\end{array}$ \\
\hline Productos alimentarios, bebidas y tabaco & 34,7 \\
Productos de la industria textil y vestuario & 67,0 \\
Cuero y productos en cuero & 66,9 \\
Madera y productos de madera (excluido los muebles) & 55,8 \\
Papel, productos de impresión e imprenta & 42,0 \\
Carbón, productos petrolíferos refinados y combustibles nucleares & 0,9 \\
Productos químicos y fibra sintética y artificial (incluidos & 26,4 \\
productos farmacéuticos) & 41,3 \\
Artículos en goma y materiales plásticos & 60,4 \\
Productos de elaboración de minerales no metalíferos & 51,0 \\
Metales y productos en metal & 51,6 \\
Máquinas y aparatos mecánicos & 28,4 \\
Máquinas eléctricas y aparatos eléctricos & 22,6 \\
Medios de transporte & 67,2 \\
Otros productos de la industria manufacturera & 46,1 \\
Total & \\
\hline
\end{tabular}

Fuente: Menghinello (2003). 
Cuadro $\mathrm{N}^{\circ} 2$

CUOTA DE MERCADO DE ITALIA SOBRE LAS EXPORTACIONES MUNDIALES Y CONTRIBUCIÓN A LAS EXPORTACIONES NACIONALES DE LOS SISTEMAS MANUFACTUREROS PYMES

\begin{tabular}{|c|c|c|c|}
\hline & $\begin{array}{c}\text { Cuota de mercado } \\
\text { de Italia sobre las } \\
\text { exportaciones } \\
\text { mundiales }\end{array}$ & $\begin{array}{c}\text { Índice de } \\
\text { especialización } \\
\text { de Italia en el } \\
\text { comercio } \\
\text { internacional }\end{array}$ & $\begin{array}{l}\text { Contribución de } \\
\text { los sistemas locales } \\
\text { manufactureros } \\
\text { Pymes a las } \\
\text { exportaciones } \\
\text { nacionales }(\%)\end{array}$ \\
\hline $\begin{array}{l}\text { Industria del azulejo y la } \\
\text { cerámica para suelos }\end{array}$ & 54,8 & 12,4 & 84,4 \\
\hline $\begin{array}{l}\text { Piedra de corte para la } \\
\text { construcción modelada y fina }\end{array}$ & 34,7 & 7,9 & 46,9 \\
\hline Cuero & 19,2 & 4,3 & 85,4 \\
\hline Calzado & 17,1 & 3,9 & 67,6 \\
\hline Muebles & 15,0 & 3,4 & 68,2 \\
\hline $\begin{array}{l}\text { Cisternas y contenedores en } \\
\text { metal, radiadores y calderas } \\
\text { para la calefacción central }\end{array}$ & 14,8 & 3,4 & 67,9 \\
\hline Tejido & 14,4 & 3,3 & 74,3 \\
\hline Bicicletas y motocicletas & 12,7 & 2,9 & 34,5 \\
\hline $\begin{array}{l}\text { Artículos de viaje, correas, } \\
\text { maletas y similares }\end{array}$ & 12,5 & 2,8 & 25,9 \\
\hline $\begin{array}{l}\text { Máquinas para la agricultura } \\
\text { y silvicultura }\end{array}$ & 11,5 & 2,6 & 84,8 \\
\hline Telas & 10,9 & 2,5 & 71,0 \\
\hline Pieles y artículos en piel & 10,8 & 2,4 & 50,3 \\
\hline Tubos & 10,7 & 2,4 & 63,3 \\
\hline Joyas y artículos de joyería & 10,4 & 2,4 & 72,0 \\
\hline $\begin{array}{l}\text { Otras máquinas de empleo } \\
\text { general }\end{array}$ & 9,6 & 2,2 & 48,1 \\
\hline $\begin{array}{l}\text { Armas, sistemas de armas } \\
\text { y municiones }\end{array}$ & 9,6 & 2,2 & 63,0 \\
\hline Bebidas & 9,5 & 2,1 & 39,9 \\
\hline $\begin{array}{l}\text { Artículos confeccionados } \\
\text { a punto }\end{array}$ & 9,3 & 2,1 & 74,1 \\
\hline Hilado de fibra textil & 8,8 & 2,0 & 70,2 \\
\hline Total & 4,4 & - & 46,1 \\
\hline
\end{tabular}

Fuente: Menghinello (2003).

Otra variable que relaciona favorablemente esta fuerte actividad industrial con las PYMES es la ocupación. Analizando los datos del primer censo industrial después de la Segunda Guerra Mundial (1951) hasta el úl- timo en el (2001), podemos visualizar y reconstruir la evolución histórica de la distribución en la ocupación según la dimensión de la empresa, estos datos se relacionan en el Cuadro $\mathrm{N}^{\circ} 3$. 


\section{Cuadro $\mathrm{N}^{\circ} 3$ \\ LA OCUPACIÓN DE LA INDUSTRIA MANUFACTURERA (EN CUOTAS SOBRE EL TOTAL) EN ITALIA SEGÚN CLASE DE DIMENSIÓN DE LA EMPRESA, DESDE 1951 HASTA EL 2001 (VALORES PORCENTUALES)}

\begin{tabular}{|c|c|c|c|c|c|c|}
\hline $\begin{array}{c}\text { Dimensión de } \\
\text { la empresa } \\
\text { según el } \\
\text { número de } \\
\text { trabajadores }\end{array}$ & $\begin{array}{c}\text { Ocupados } \\
1951\end{array}$ & $\begin{array}{c}\text { Ocupados } \\
1961^{*}\end{array}$ & $\begin{array}{c}\text { Ocupados } \\
1971\end{array}$ & $\begin{array}{c}\text { Ocupados } \\
1981\end{array}$ & $\begin{array}{c}\text { Ocupados } \\
1991\end{array}$ & $\begin{array}{c}\text { Ocupados } \\
2001\end{array}$ \\
\hline $1-2$ & & & & & \\
$3-5$ & 17,1 & - & 7,5 & 7,3 & 6,9 & 7,6 \\
$6-9$ & 8,6 & - & 6,4 & 7,3 & 8,8 & 8,1 \\
$10-15$ & 5,0 & - & 5,2 & 7,0 & 8,7 & 8,5 \\
$16-19$ & - & - & 5,3 & 8,3 & 9,7 & 10,2 \\
$20-49$ & - & - & 2,6 & 3,6 & 5,0 & 5,2 \\
$50-99$ & - & - & 11,8 & 12,7 & 15,3 & 16,3 \\
De 10 a 99 & 19,8 & - & 9,5 & 9,1 & 8,9 & 10,1 \\
$100-199$ & - & - & 29,3 & 33,6 & 38,9 & 41,8 \\
$200-249$ & - & - & 9,2 & 9,0 & 8,1 & 8,7 \\
$250-499$ & - & - & 7,6 & 2,7 & 2,4 & 2,4 \\
$500-999$ & - & - & 6,8 & 6,1 & 6,4 & 6,5 \\
De 100 a 999 & 23,0 & - & 26,3 & 24,7 & 21,9 & 22,6 \\
1.000 y más & 26,6 & - & 25,3 & 20,1 & 14,8 & 11,4 \\
Total & 100,0 & - & 100,0 & 100,0 & 100,0 & 100,0 \\
\hline
\end{tabular}

*Los datos para el censo de 1961 no están disponibles.

Fuente: Elaboración propia a partir de censos industriales del ITSAT.

Los datos reflejan cómo la ocupación ha estado creciendo de forma constante en aquellas empresas con menos de 50 trabajadores desde 1951 hasta el último censo industrial, reafirmando así la importancia de las PYMES en el sistema económico italiano.

\section{Los distritos industriales italianos en la actualidad}

A partir de los años noventa, época que coincide con el boom de la globalización y con ello el acortamiento de las distancias entre los mercados producto de las TIC (tecnologías de la información y la comunicación), los distritos industriales italianos han debido hacer frente a nuevos competidores, que particularmente tienen un coste salarial notablemente inferior. Esta situación ha llevado a muchas empresas a deslocalizar parte de su producción en la búsqueda de menores costes y así obtener mayores beneficios. Estas estrategias han ido progresivamente interrumpiendo las relaciones entre las empresas locales y la difusión del conocimiento, afectando así a este círculo virtuoso de cooperación tan característico del modelo en sus años de esplendor, situación que ha ido debilitando la capacidad productiva del área (Garofoli, 2003), reduciendo significativamente el número de empresas en los sectores del made in Italy.

La deslocalización ha venido a través de la apertura de nuevas filiales mediante la firma de contratos con subproveedores concesionados a proveedores extranjeros. Estos 
países han sido asiáticos y de Europa del Este, principalmente Rumania y en medida inferior, países del Mediterráneo.

La respuesta a este desafío externo ha determinado, en algunos casos, la estrategia de la diversificación productiva ${ }^{11}$. Esta estrategia ha estado seguida por aquellos distritos que han preferido la vía de la introducción de productos diferenciados y de mayor calidad para evitar la desventaja de costes laborales y de producción. En tales estrategias se acompaña el objetivo de una mayor autonomía sobre el mercado ${ }^{12}$.

La idea fue entonces entrar en nichos más específicos y poder así alargar su mercado meta a través de los beneficios de las economías de alcance, otorgando por ejemplo productos intermedios a otros sectores productivos, sobre la base de un conocimiento técnico ya instaurado por largo tiempo dentro del distrito.

\section{Políticas de intervención para el sostenimiento y competitividad de los distritos; una mirada hacia el futuro}

En los sistemas de pequeñas empresas se debe aumentar la atención a los siguientes argumentos con el objetivo de reforzar

\footnotetext{
${ }^{11}$ Esta situación también se pudo comprobar en terreno en el distrito textil de Como. A través de entrevistas a empresarios se pudo constatar un mejor aprovechamiento de la maquinaria ya existente en las plantas, en este caso de alto nivel tecnológico, diversificando la producción y dándole mayor funcionalidad a los implantes productivos con el objeto (según señalaban los empresarios) de poder hacer frente a la áspera competencia China e India.

12 En las visitas a las empresas de la materia plástica de Varese los entrevistados indicaron que las empresas finales eran las que estaban en contacto directo con el mercado, ejerciendo el poder sobre la producción de las empresas intermedias en concordancia con las necesidades que establecían los clientes finales. Por tanto, el sistema Varesino presenta características de ser más bien un cluster que un distrito, esto debido a la producción de un bien más estandarizado, lo que implica menor división en el trabajo, y un menor contacto con el mercado final, característica innata de los distritos con sus métodos de producción flexible.
}

aquellas capacidades adquiridas en los años de mayor éxito y desarrollo económico. Según Garofoli (2003), esto sería:

- Capacidad de anticipación ante el cambio y las contingencias, esto conlleva un rol activo en las decisiones que se tomen en los métodos de producción, de los productos a introducir en el mercado $y$, por tanto, del posicionamiento estratégico que se hará de los mismos;

- Introducción de una cultura estratégica en una lógica de programación de objetivos y resultados a partir de la utilización de recursos alternativos en una suerte de revivir. Para las PYMES se trata se la tradicional interacción estructuraconducta-performance; e

- Introducción progresiva de una cultura de leadership, en vez de mantener un comportamiento de follower.

Una de las herramientas para afrontar lo anteriormente expuesto con una buena posibilidad de éxito es ampliando las ventajas de las PYMES, utilizando la red interna en el sistema local, así como las redes externas que consistan en internalizar conocimiento proveniente del exterior, actuando como technological gatekeepers. Estas inversiones para el reforzamiento de las "economías externas" no pueden ser financiadas exclusivamente por los operadores públicos o las instituciones non-profit; es oportuno que sean efectuadas por las mismas empresas que conforman el sistema productivo con el fin de mejorar su posición competitiva, por un lado, y para el reforzamiento de la "eficiencia colectiva" del sistema local, por el otro (Schmitz, 1999).

\section{Consideraciones finales}

El estudio de los distritos industriales italianos requiere una cuantificación empírica más formalizada; es frecuente la ambigüedad de algunos estudios al respecto. Sin embargo, existe un hecho que es indudable y que pudo ser constatado en este artículo: los sistemas productivos locales italianos, en los cuales tienen un rol fundamental la pequeña y mediana empresa, absorben en Italia una cuota de ocupación en la industria manufacturera que va desde el $35 \%$ al $40 \%$ del total. 
Con respecto a las ventajas derivadas de las economías externas, esto es, el pool de habilidades y de mano de obra, se crea una especialización por parte del trabajador que permanece en el área, lo que crea un soporte especializado en imputs, como en servicios dentro del territorio y spillovers tecnológicos que efectivamente ayudan a la difusión de nuevas innovaciones, las cuales vienen introducidas, generalmente, por las grandes empresas. Sin embargo, las instituciones en su papel de garantizar la viabilidad del modelo parecen estar perplejas ante la falta de iniciativa para el desarrollo y sustento de los distritos.

De esta reflexión surge la necesidad de una acción conjunta por parte de empresarios e instituciones para una adecuada política industrial que permita un camino de modo sistemático y organizado hacia el mercado. En el mundo de hoy es necesaria la búsqueda de servicios avanzados, intangibles y estratégicos que favorezcan las condiciones para la absorción de conocimiento en el desarrollo de nuevas innovaciones. Bajo estas condiciones los distritos industriales y sistemas productivos locales, serán capaces de anticipar las contingencias externas $y$, por tanto, mantener el control en sus procesos de transformación.

\section{Referencias bibliográficas}

ARRIGHETTI, A. y SERAVALLI, G., Istituzioni e dualismo dimensionale nell 'industria italiana'. In: BARCA, F. Storia del capitalismo italiano dal 1945 ad oggi. Roma: Donzelli, 1997.

BECATTINI, G. Dal "settore" industriale al "distretto" industriale. Alcune considerazioni sull'unità di indagine dell'economia industriale. Rivista di economia e politica industriale, 1979, Vol. 5, No 1, p. 7-21.

BECATTINI, G. Il distretto industriale, un nuovo modo di interpretare il cambiamento economico. Torino: Rosenberg \& Sellier, 2000.

BECATTINI, G. Del distrito industrial Marshalliano a la teoría del distrito contemporánea, una breve reconstrucción crítica. Investigaciones Regionales. Asociación española de ciencia regional, 2002, № 1, p. 9-32.
BECATTINI, G.; BELLANDI, M.; DEI OTTATI, G. e SFORZI, F. (a cura di). II caleidoscopio dello sviluppo locale. Trasformazioni economiche nell'Italia contemporanea. Torino: Rosenberg \& Sellier, 2001.

BELLANDI, M. El distrito industrial en Alfred Marshall. Estudios Territoriales, 1986, No 20, p. 31-44.

BIRCH, D. The job creation process. MIT, Program on Neighborhood and Regional Change. Cambridge: Mass, 1979.

FUÀ, G. L'industrializzazione nel Nord Est e nel Centro. In: FUÀ, G. e ZACCHIA, C. (a cura di). Industrializzazione senza fracture. Bologna: Il Mulino, 1983, p. 22.

FUÀ, G. Small-scale industry in rural areas: the Italian Experience, en The Balance between Industry and Agriculture in Economic Development. London: Ed. K.J. Arrow, Macmillan, 1988.

GAROFOLI, G. Modelli locali di sviluppo. Milano: Franco Angeli, 1991.

GAROFOLI, G. (a cura di). Impresa e territorio. Bologna: Il Mulino, 2003.

GIARDELLO, M. Cultura e sviluppo locale nelle piccole comunità, Roma: Ed. Rubbetino, 2006.

GUDGIN, G. Industrial Location Processes and Regional Employment Growth, Farnborough: Saxon House, 1978.

ITSAT - ISTITUTO NAZIONALE DI STATISTICA. Censimenti italiano. Roma: ITSAT, varios años. Disponible en Internet: http:// www.istat.it/

MARKUSEN, A. Profit Cycles, Oligopoly and Regional Development. Cambridge, MA: MIT Press, 1985.

MARSHALL, A. Industry and Trade. London: Macmillan, 1919.

MARSHALL, A. Principles of Economics. London: Macmillan and Co. Malinowski, 1961. 
MASKELL, P. Social capital, innovation, and competitiveness. In: BARON, S.; FIELD, J. \& SCHULLER, T. (Eds.). Social capital. Oxford: Oxford University Press, 2000, p. 110-123.

MENGHINELLO, S. Dimensione locale e competitività sui mercato internazionali:il contributo dei sistemi locali di piccola e media impresa alle esportazioni nazionali. Roma: Dipartimento Statistiche economiche ISTAT, 2003.

NORTH, D. Institutional change: A Framework of Analysis. Economic History, EconWPA, 1994, No 9412001, 1994.
PIORE, M. \& SABEL, C. The Second Industrial Divide, Possibilities for prosperity, New York: BasicBook, 1984.

SCHMITZ, H. Collective Efficiency and Increasing Returns. Cambridge Journal of Economics, 1999, Vol. 23, p. 465-483.

VÁZQUEZ BARQUERO, A. Desarrollo local: una estrategia de creación de empleo. Madrid: Editorial Pirámide, 1988. 
